\title{
Active Target TPC equipped with GEM for the AMADEUS experiment
}

\section{Poli Lener* Laboratori Nazionali di Frascati - INFN, Frascati, Italy \\ E-mail: marco.polilenerelnf.infn.it}

\section{G. Corradi, C. Curceanu, E. Sbardella, D. Tagnani}

Laboratori Nazionali di Frascati - INFN, Frascati, Italy

\begin{abstract}
In this paper we report the R\&D activity on a new GEM-based TPC detector for the inner region of the AMADEUS experiment, which is aiming to perform measurements of the low-energy negative kaon interactions in nuclei at the DAФNE $\mathrm{e}^{+} \mathrm{e}^{-}$collider at LNF-INFN.

A novel idea of using a GEM-TPC as a low mass target and detector at the same time comes motivated by the need of studying the low energy interactions of $\mathrm{K}^{-}$with nuclei in a complete way, tracking and identifying all of the produced particles.

Even more, what makes revolutionary the experimental proposal is the possibility of using different gaseous targets without any other substantial intervention on the experimental setup, making it a flexible multipurpose device. This new detection technique applied to the nuclear physics requires the use of low-mass material and very pure gases such as Hydrogen, Deuterium, Helium-3, Helium-4, etc.

Encouraging measurements have demonstrated the possibility to reach a detector gain up to $10^{4}$ without damages and instabilities with a GEM-TPC filled with a pure helium gas. The use of Hydrogen and Deuterium will be investigated in the near future.
\end{abstract}

52 International Winter Meeting on Nuclear Physics - Bormio 2014,

27-31 January 2014

Bormio, Italy

\footnotetext{
* Speaker.
} 


\section{Introduction}

An important, yet unsolved problem, in hadron physics is how the hadron masses and interactions change in the nuclear medium. This topic could be investigated by means of "inmedium hadron-mass spectroscopy", producing bound states, of a hadron by which to deduce the hadron-nucleus potential and the in-medium hadron masses. The AMADEUS (Antikaon Matter At DAФNE Experiments with Unraveling Spectroscopy) experiment [1],[2] will study the low energy interactions of negative kaons with nucleons and nuclei. The dedicated AMADEUS setup will be implemented inside the KLOE [3] Drift Chamber (DC), in the free space between the beam pipe and the DC entrance wall. Currently, the main component of the experimental setup to be developed is an active target GEM-based [5] TPC, which will act as low mass target and particles tracking detector at the same time. A drawing of the dedicated AMADEUS setup surrounding the beam pipe within KLOE detector is given in Fig. 1.
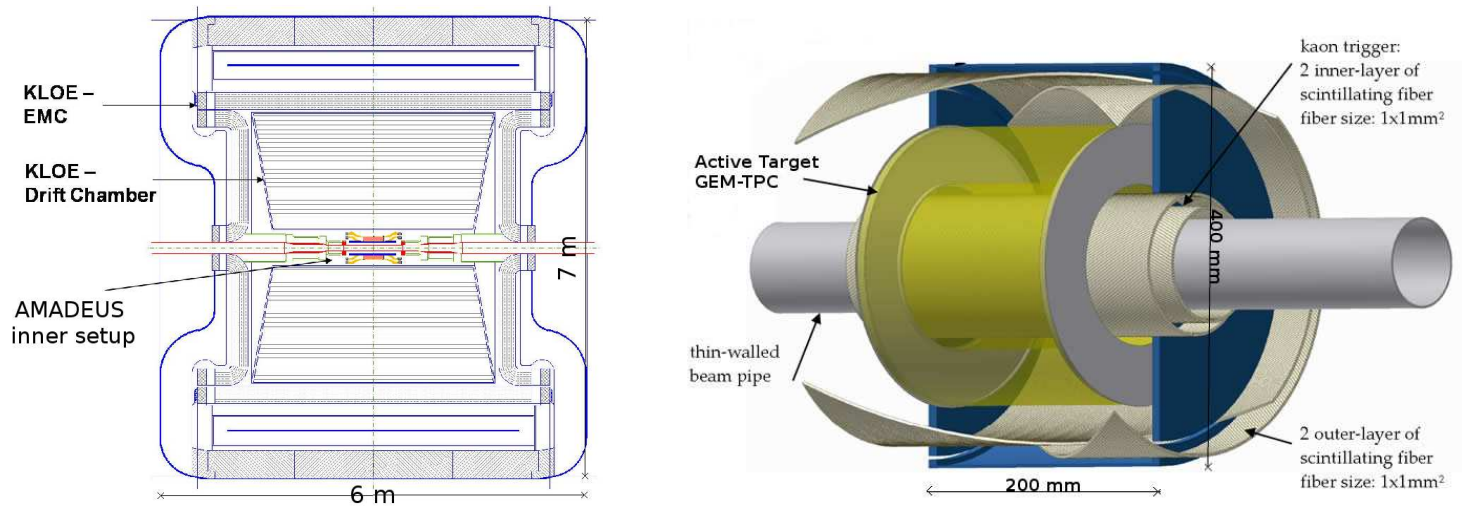

Figure 1: Cross-section of the KLOE detector including the AMADEUS inner setup inside the Drift Chamber (left) and the inner dedicated setup of AMADEUS. From the beam pipe to the outer region: the Kaonic Trigger, the active target GEM based-TPC.

The GEM-TPC in AMADEUS will be $20 \mathrm{~cm}$ long with an inner diameter of $8 \mathrm{~cm}$ and an outer one of $40 \mathrm{~cm}$. The main requirements can be summarized as:

- a spatial resolution better than $200 \mu \mathrm{m}$ in $\mathrm{X}-\mathrm{Y}$ and $500 \mu \mathrm{m}$ in Z;

- a detector material budget lower than $0.5 \%$ of $\mathrm{X}_{0}$;

- a rate capability of $\sim 5 \mathrm{kHz} / \mathrm{cm}^{2}[6]$;

Since most of the above requirements are easily fulfilled by a GEM-TPC with a standard gas mixture, the R\&D activity at the Laboratori Nazionali di Frascati is mainly focused on the study of the detector performances with pure gas.

A GEM-based TPC prototype of $10 \times 10 \mathrm{~cm}^{2}$ with a drift gap of $15 \mathrm{~cm}$ was realized and tested both in laboratory and at the Paul Scherrer Institute (PSI). Details of the prototype construction and of the test beam setup can be found in Ref. [7].

In Sec. 2 preliminary simulation studies on an active hydrogen target GEM-TPC in the AMADEUS 
experiment are shown, while in Sec. 3 the detector performances obtained at the $\pi \mathrm{M} 1$ beam facility, in terms of detector efficiency and spatial resolution, are presented.

\section{Simulation for a hydrogen GEM-TPC}

Simulation studies have been performed with GARFIELD and GEANT-4 programs in order to investigate the use of an active target GEM-TPC as the inner tracker of the AMADEUS experiment. The GEM-TPC is constrained to fit into the free volume between the DAФNE beam-pipe and the KLOE drift chamber. In the GEANT-4 simulation we set the following conditions:

- the phase space volume of $\Phi$ mesons is populated according to the recently proposed scheme of crab-waist collisions for DAФNE;

- the elastic scattering process $\mathrm{K}^{\mp} \mathrm{p}->\mathrm{K}^{\mp} \mathrm{p}$ as an example;

- the charged particles undergo multiple scattering throughout the gas contained inside GEMTPC, losing energy only by ionization;

- the particles move inside a magnetic field of $\mathrm{B}=0.5 \mathrm{~T}$.

A possible configuration of the active target GEM-TPC detector is sketched in Fig. 2.

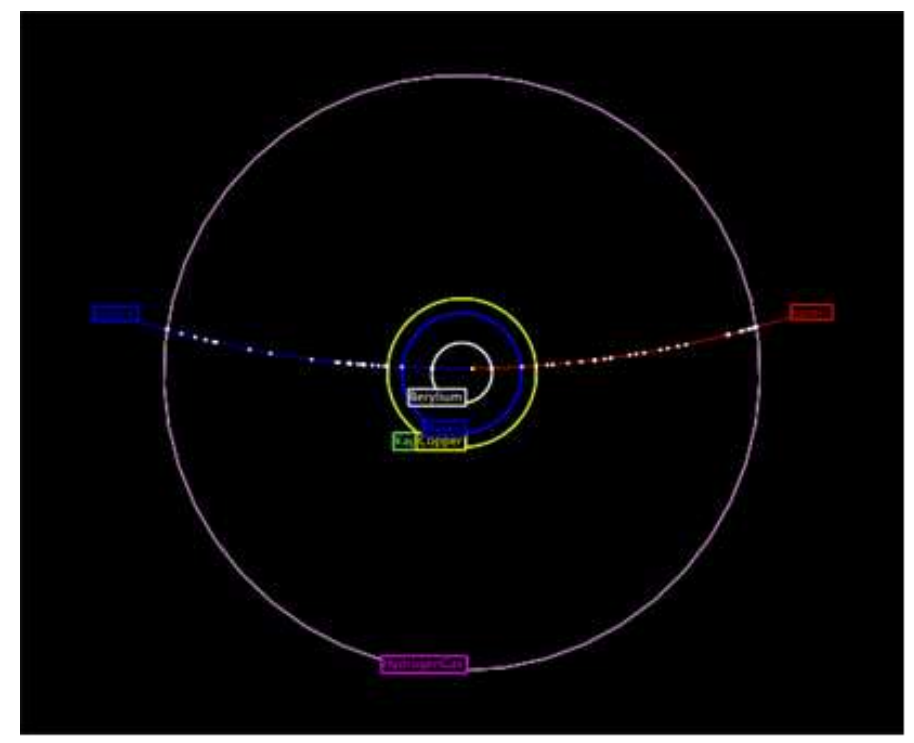

Figure 2: GEANT simulation of the inner setup of the AMADEUS experiment.

It has a cylindrical geometry along the Z-axis, which matches the DAPhiNE $\mathrm{e}^{+} \mathrm{e}^{-}$beams directions. The inner layer is the beam-pipe, a beryllium foil of $2 \mathrm{~cm}$ diameter and $0.5 \mathrm{~mm}$ thickness. The beam-pipe layer is followed by a $0.5 \mathrm{~mm}$ thick segmented detector made of plastic scintillator for a fast-trigger of kaons produced by the $\Phi$ decay. Then the setup contains an active target GEMTPC, consisting in two $100 \mu \mathrm{m}$ thick kapton foils, copper clad on each side ( $5 \mu \mathrm{m}$ thick) which defines the field cage of the chamber.

For this simulation, the detector is filled with pure Hydrogen gas at standard condition $\left(300{ }^{\circ} \mathrm{K}, 1\right.$ 
atm).

The crab-waist configuration of the DAФNE collider makes the crossing angle between the $\mathrm{e}^{+} \mathrm{e}^{-}$ beams to span $25 \mathrm{mrad}$, which causes a momentum spread of about $15 \mathrm{MeV} / \mathrm{c}$. Accordingly, the resulting kaons are emitted with a momentum spread which ranges between 115 and $141 \mathrm{MeV} / \mathrm{c}$. As an example of this study, we consider the $\mathrm{K} \mathrm{p}->\mathrm{K}$ p elastic scattering with a $127 \mathrm{MeV} / \mathrm{c}$ incident kaon. The momenta of the scattered kaon range from $\sim 40$ to $127 \mathrm{MeV} / \mathrm{c}$ and the proton momenta can be as high as $\sim 165 \mathrm{MeV} / \mathrm{c}$ as shown in Fig. 3. This figure also shows that kaons (blue line) of momenta larger than $100 \mathrm{MeV} / \mathrm{c}$ are emitted at forward angles along with protons (red line), otherwise for kaon of momenta lower than $100 \mathrm{MeV} / \mathrm{c}$, proton and kaon are emitted backward. Therefore only an active target TPC-GEM allows to track the considered elastic scattering in both forward and backward regions.

In Fig. 3 is shown the energy loss by ionization as a function of the particles momenta considered in the elastic scattering. It should be noted the high separation power in the particle identification for kaons and protons. We also reported the number of cluster per mm due to Hydrogen gas primary ionization. At very low momenta the discrepancy between GARFIELD and GEANT-4 is to be assigned to the clustering model invoked in GARFIELD, which fails at low $\beta \gamma$.
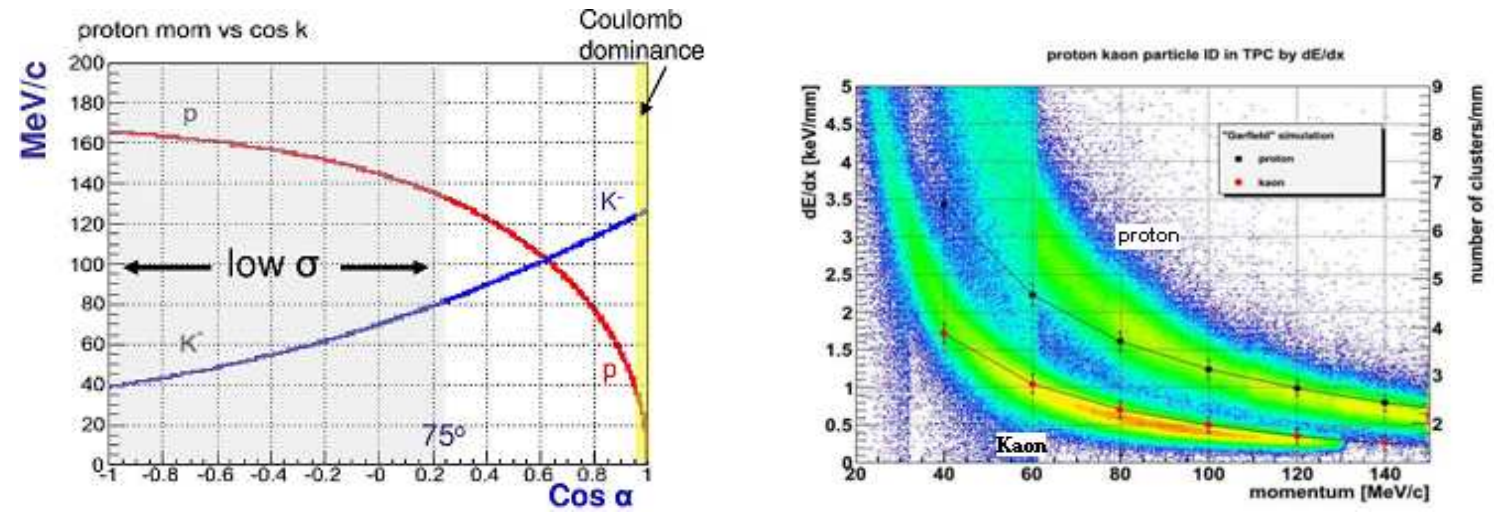

Figure 3: Kinematics of the $\mathrm{K}^{-} \mathrm{p}$ elastic scattering: the blue curve shows the momentum of the emitted kaon versus the cosine of the angle between the kaon direction and the beam direction. The red curve shows the kinematics of the associated proton (left). Energy loss (GEANT) and primary ionization (GARFIELD) as a function of the particle momentum (right).

We also evaluated the effect of the multiple Coulomb scattering when a low momentum kaon crosses the Hydrogen gas target. In order to study this effect, a $127 \mathrm{MeV} / \mathrm{c}$ pencil beam of kaons is sent through the gas target and the angular distribution of deflected kaons is evaluated at the outer field cage wall. At about $200 \mathrm{~mm}$ far from the $\Phi$ production point, the angular distribution is described for both the $\mathrm{x}$ - and $\mathrm{y}$-coordinates by a Gaussian distribution with a $\sigma$ of $\sim 85 \mu \mathrm{m}$ (Fig. 4). This means that the elastic differential cross section can be derived with an uncertainty of about 1 mrad (FWHM). The most important gas properties are simulated with GARFIELD program and compared to the standard $\mathrm{Ar} / \mathrm{CO}_{2}$ (70/30) gas mixtures. The Hydrogen gas is also simulated at different temperatures $\left(50{ }^{\circ} \mathrm{K}\right.$ to $450{ }^{\circ} \mathrm{K}$ in step of $100{ }^{\circ} \mathrm{K}$ ) and different pressures ( 1 atm to $10 \mathrm{~atm}$ in step of $1 \mathrm{~atm})$, which will be used in future simulation studies. 

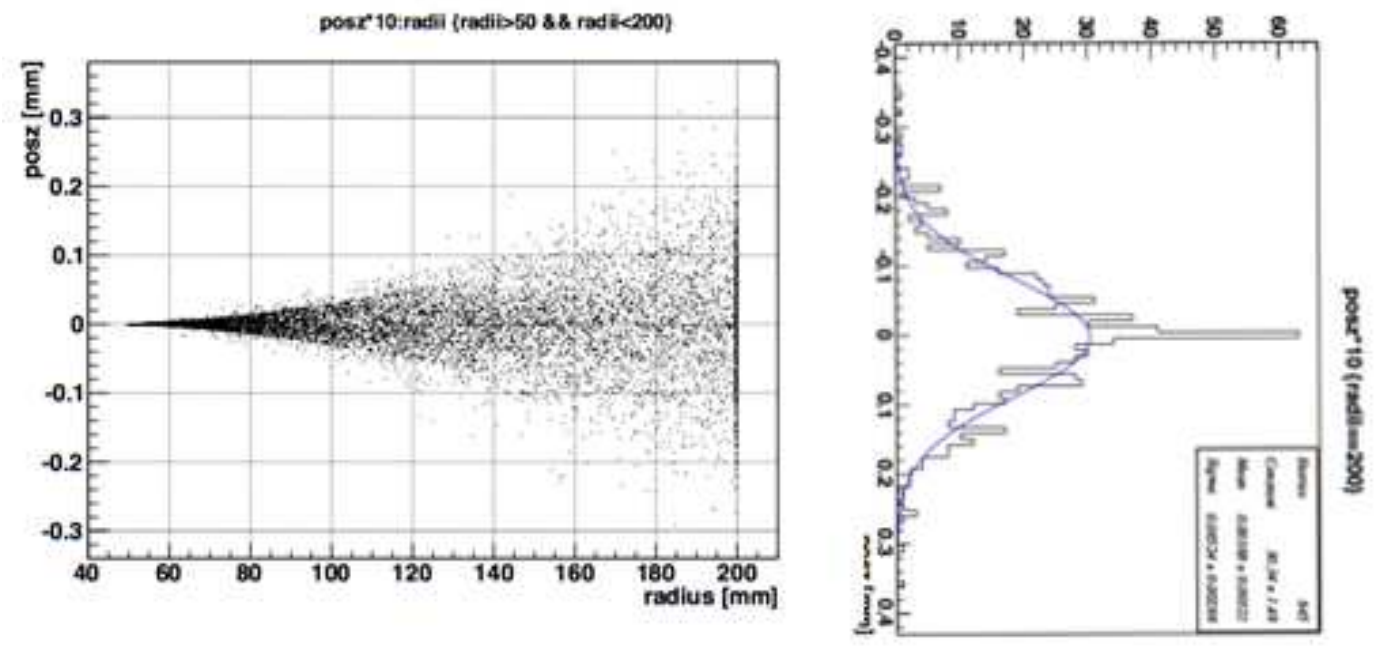

Figure 4: Multiple Coulomb scattering in the Hydrogen gas (left) and the relative angular distribution (right) for an incident $127 \mathrm{MeV} / \mathrm{c}$ kaon.

\begin{tabular}{cccc}
\hline \hline & $\begin{array}{c}\text { Drift Velocity } \\
\text { at } 100 \mathrm{~V} / \mathrm{cm} \\
(\mathrm{cm} / \mu \mathrm{s})\end{array}$ & $\begin{array}{c}\text { Tran. Diffusion } \\
\text { at } 100 \mathrm{~V} / \mathrm{cm} \\
(\mu \mathrm{m} / \sqrt{\mathrm{cm}})\end{array}$ & $\begin{array}{c}\text { Long. Diffusion } \\
\text { at } 100 \mathrm{~V} / \mathrm{cm} \\
(\mu \mathrm{m} / \sqrt{\mathrm{cm}})\end{array}$ \\
\hline Hydrogen & 0.39 & 322 & 253 \\
Deuterium & 0.37 & 331 & 250 \\
Helium-3 & 0.35 & 571 & 391 \\
Helium-4 & 0.32 & 601 & 414 \\
$\mathrm{Ar} / \mathrm{CO}_{2}=70 / 30$ & 0.23 & 242 & 237 \\
\hline
\end{tabular}

Table 1: Main gas properties. The standard $\mathrm{Ar} / \mathrm{CO}_{2}$ gas mixture is reported for comparison.

\section{GEM-TPC measurements and performances}

\subsection{Gas Gain Measurement in Laboratory}

The effective gain of a triple-GEM detector has been measured for different gas mixtures and for pure helium using a high intensity $5.9 \mathrm{keV}$ X-ray tube. Effective gain value was obtained from the ratio of the pad current with high voltage on the GEM foils, to the current on the first GEM, with no high voltage on the GEM foils, as shown in Fig. 5.

Detector instabilities have been observed only for the prototype filled with a pure helium gas at a gain greater than $\sim 3 \times 10^{4}$.

\subsection{GEM-TPC performances at the PSI beam facility}

The performances of the GEM-TPC prototype have been studied at the $\pi \mathrm{M} 1$ beam facility of the PSI without magnetic field. The $\pi \mathrm{M} 1$ beam is a quasi-continuous high-intensity secondary beam providing hadrons with a momentum resolution of $\sim 1 \%$. The measurement of the detector 


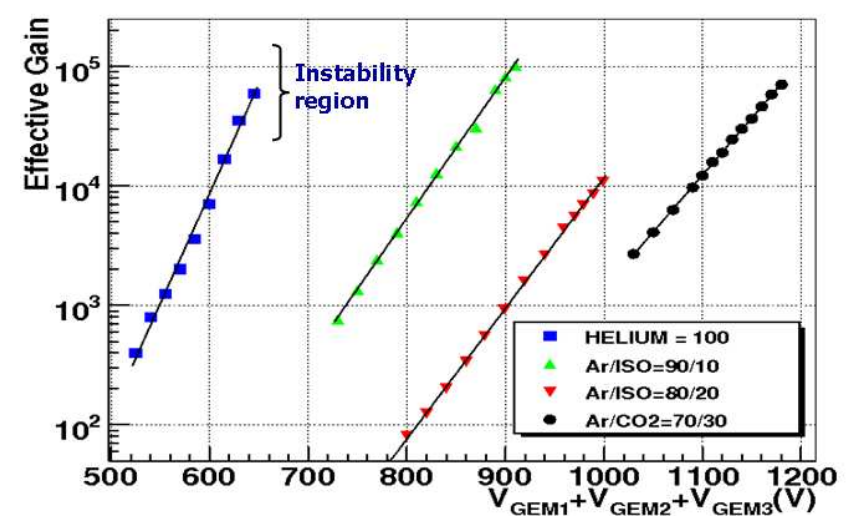

Figure 5: Effective gain as a function of the sum of the voltages applied on the three GEM foils for different gas mixtures and pure helium gas. The curve of the $\mathrm{Ar} / \mathrm{CO} 2=70 / 30$ gas mixture is reported for comparison.

performances has been performed with a beam rate of $\sim 200 \mathrm{~Hz}$. The prototype readout was composed by 4 columns of 32 pads of $3 \times 3 \mathrm{~mm}^{2}$ each. Each pad was connected to a front-end board based on CARIOCA-GEM chip [8]. The discriminator threshold on the front-end electronics was set to $\sim 2.1 \mathrm{fC}$.

The single pad row detection efficiency has been evaluated considering the fraction of the hits in a single pad row with respect to a selected track, while the spatial resolution has been evaluated by the residuals between the hit position in a single pad row, excluding it from the fit track, and the interpolated hit position from the remaining single pad rows for each selected track.

The detector efficiency and the spatial resolution in the drift direction for protons of $440 \mathrm{MeV} / \mathrm{c}$ as a function of the gas gain are shown in Fig. 6. A detection efficiency of $\sim 95 \%$ and a spatial resolution along the drift direction of $350 \mu \mathrm{m}$ have been achieved with a pure noble Helium gas.

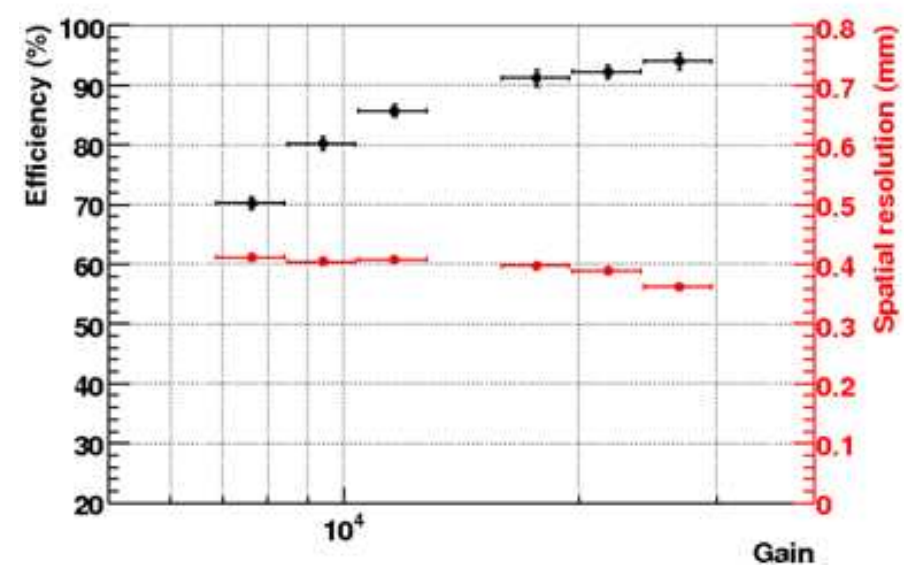

Figure 6: Efficiency (black points) and spatial resolution (red points) in the drift direction for protons of $440 \mathrm{MeV} / \mathrm{c}$ as a function of the detector gain with a pure Helium gas and a front-end electronic threshold of $\sim 2.1 \mathrm{fC}$. 


\section{Conclusions}

The R\&D activity on a new active target GEM-based TPC for the inner region of the AMADEUS setup has started at the Laboratori Nazionali di Frascati (INFN). A GEM-TPC prototype with a drift gap of $15 \mathrm{~cm}$ has been successfully built and tested at the $\pi \mathrm{M} 1$ beam facility of the Paul Scherrer Institute with low momentum proton beam and filled pure helium gas.

The detector performances have shown a detection efficiency of $95 \%$ and a spatial resolution of $350 \mu \mathrm{m}$ showing the possibility to use such TPC as a low mass target and detector at the same time in the AMADEUS environment.

Moreover, the achieved results open the way of the use of pure Hydrogen and Deuterium gases at ambient and cryogenic temperatures, which will be investigated in future test beams.

\section{Acknowledgments}

The authors would like to thank the coordinator of the PSI beam lines, Dr. Konrad Deiters, for the excellent cooperation and support.

Part of this work was supported by the European Community-Research Infrastructure Integrating Activity "Study of Strongly Interacting Matter" HadronPhysics 2 (HP2), Grant Agreement No. 227431, and HadronPhysics 3 (HP3), Contract No. 283286, under the Seventh Framework Programme of EU.

\section{References}

[1] AMADEUS Collaboration, AMADEUS Letter of Intent, http://www.Inf.infn.it/Infadmin/direzione/roadmap/LOI_MARCH_AMADEUS.pdf.

[2] AMADEUS Collaboration, AMADEUS Phase-1: Setup and Roll-in Proposal, LNF-07/24(IR) 2007.

[3] D. Adinolfi et al., Nucl. Instr. and Meth. A 461 (2001) 25.

[4] M. Bazzi et al., Nucl. Instr. and Meth. A 671 (2012) 125.

[5] F. Sauli, Nucl. Instr. and Meth. A 386 (1997) 531.

[6] M. Poli Lener, Triple-GEM detectors for the innermost region of the muon apparatus at the LHCb experiment, Ph.D Thesis, CERN-THESIS-2006-13.

[7] M. Poli Lener et al., Performances of a GEM-based Time Projection Chamber prototype for the AMADEUS experiment, arXiv:1302.3054 [physics.ins-det].

[8] A. Alfonsi et al., IEEE NSS MIC Vol. 6 (2007) 4671. 